\title{
The design and development of a smart fire-fighter robotic system
}

\begin{abstract}
Fire fighters extinguish fires to protect lives and to avoid destruction of property and environment. Through extinguishing operation, many firefighters are killed and injured due to the absence of information about hazard building infrastructure. In this paper, we design and implement a prototype robotic system which intends to assist the firefighters in accomplishing their duties, including navigation, rescue, and obtain localization information. In addition, the robotic system will navigate the firefighters through the safest route inside the building where GPS signals are inaccessible.
\end{abstract}

Volume 3 Issue 6 - 2017

\author{
Saleh Alhawas, Mohamad Sabha,Tareq \\ Alhmiedat \\ Department of Information Technology, Tabuk University, Saudi \\ Arabia
}

Correspondence: Tareq Alhmiedat, Department of
Information Technology, Tabuk University, Saudi Arabia Email t.alhmiedat@ut.edu.sa

Received: April 22, 2017| Published: December 08, 2017

\section{Introduction}

Fire fighting and rescue activity are considered as very dangerous missions. Injured firefighters and civilians inside hazard buildings are risky to rescue and evacuate. Sometimes, firefighters face serious challenges to get in the hazard building to extinguish fire, and locate the civilians, because in most cases, firefighters do not have a prior knowledge about the hazard building infrastructure. Firefighters face serious risks on the job, where they face flames, heat, high level of $\mathrm{CO}$ or $\mathrm{CO}_{2}$, and physical and mental stress, A large number of firefighters have been injured during practicing their duties, including extinguishing fire, and rescuing civilians. According to Schildt, ${ }^{1} 22$ firefighters were died in during fire grounds operations in 2012, and an estimated 81,070 fire-fighter injuries occur irregularly in the US. Therefore, there is an ideal target for robot technology to keep away firefighters from danger. Robotics is the branch of mechanical engineering, electrical engineering and computer science that deals with the design, construction, operation, and application of robots, as well as computer systems for their control, sensory feedback, and information processing. These technologies deal with automated machines that can take the place of humans in dangerous environments or manufacturing processes, or resemble humans in appearance, behaviour, and or cognition. Many of today's robots are inspired by nature contributing to the field of bio-inspired robotics. ${ }^{2}$ Rescue robotics has been suggested by DARPA study as an application domain for the research in human robot integration. ${ }^{3}$ This paper presents a prototype robotic system designed to navigate firefighters in unknown terrain, and determine the location of civilians inside the hazard building. Moreover, the designed robotic system is able to find the shortest and the safest route to civilians indoors where GPS signals are inaccessible indoors. The rest of this paper is organized as follows: Section 2 reviews the most significant relevant works. In Section 3, the system design is presented and discussed. The system implementation and results obtained from real experiments are presented in Section 4. And finally, Section 5 shows a conclusion and future works.

\section{Related work}

In this section, the existing works are discussed. One of the most significant relevant works is "Rescue me, Robot", a robot machine that aimed to reduce the exposure of human firefighters to extreme fires. ${ }^{4}$ Also, US Navy unveiled its latest prototype robot named Humanoid robot, which is a 5 -foot and 10 two-legged robots which can see through dense smoke and carry a house on its own. ${ }^{5}$ Ballam is an autonomous fire-fighter machine, where its mission is to follow firefighters into battle, carrying stuff that either too heavy or to offer personnel break from backpack and equipment toting. ${ }^{6}$ On the other hand, researchers at MIT in Cambridge, working on the fire-fighter robot called Atlas. ${ }^{7}$ This research project is ongoing and aims to design and implement a robot machine that can walk into dangerous places and do something useful there (for instance rescue civilians). As noticed above, most of the proposed systems suffer from either the design complexity or high cost. This work aims to design and implement a low-cost prototype robotic system which will be able to navigate firefighters to a safe root, and to localize civilians' positions indoors.

\section{System design}

In this section, the prototype robotic system is presented, in which it consists of several sensors used for different purposes, servo motors, processor, and communication module for exchanging data between the fire-fighter and the robotic system. The prototype robotic system architecture is depicted in Figure1. The presented system consists of two parts: the robotic unit and the remote-control unit. The robotic unit carries out 4 main functions as follows: First, robotic unit navigates the terrain using inertial navigation system and distance sensors, and creates a virtual map based on the navigation information. Second, robotic unit senses the surrounding environment while moving from one point to another (for instance, the level of temperature). Third, robotic unit can detect the presence of civilians in hazard areas, using both mice and motion sensors. And Fourth, robotic unit sends the navigation and sensing information to the remote access unit, in order to share this information with firefighters. On the other hand, the remote-control unit receives the navigation and sensing information from robotic unit, builds a database about the environment (routes, environment conditions, and position of civilians), and guides the firefighters to the shortest and safest route to civilians. The robotic unit communicates with the remote access unit through Wi-Fi network. Figure 2 presents the robotic system flowchart. 

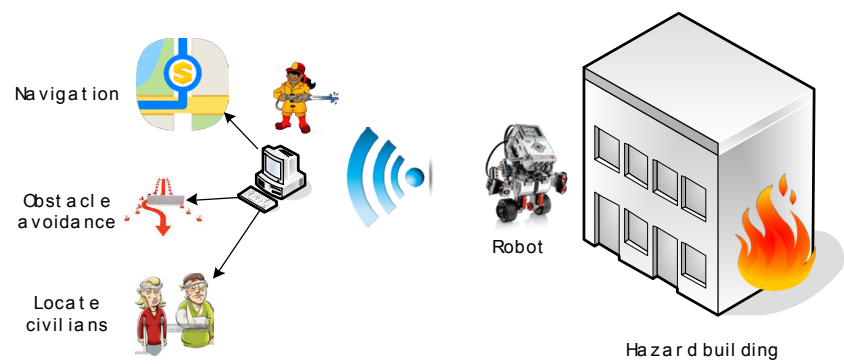

Figure I Robotic system concept.

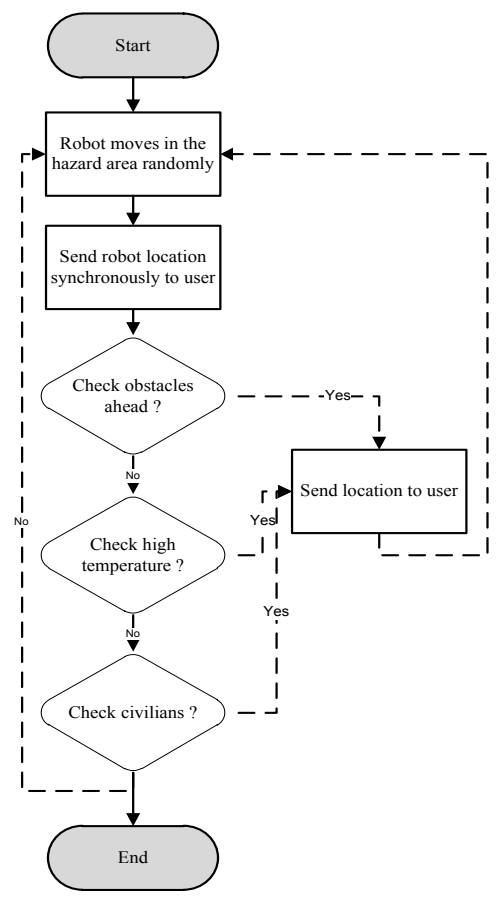

Figure 2 Robotic system flowchart.

\section{Experimental results}

This section presents the hardware employed to implement the proposed robotic system. In addition to presenting a number of real experiments which have been carried out to assess the efficiency of the proposed system.

\section{Hardware architecture}

The designed system consists of two parts: robot and remotecontrol unit, as presented in Figure 3. The robot is able to navigate the terrain, and transmit the obtained information from onboard sensors to the remote-control unit where the data is processed. For implementation purposes, Lego EV3 development kit presented in Figure 4, has been employed to implement the prototype robotic system. Lego EV3 development kit has been employed by many researches to design and implement many prototype robotic systems to proof a concept..$^{8-11}$

The designed prototype robotic system is shown in Figure 5, which consists of 4 different sensors, as follows:
Infrared sensor: this determines the existence of obstacles facing the robot while navigating the terrain.

Motion sensor: any movement faces the robot can be detected and recorded by the motion sensor.

Temperature sensor: the temperature level is sensed in the hazard building, and fire-fighter will get full information about the location and level of temperature for each risky location.

Sound sensor: any sounds can be detected by the sound sensor attached to the robotic system, in order to detect any injured or stuck civilian in the hazard building.

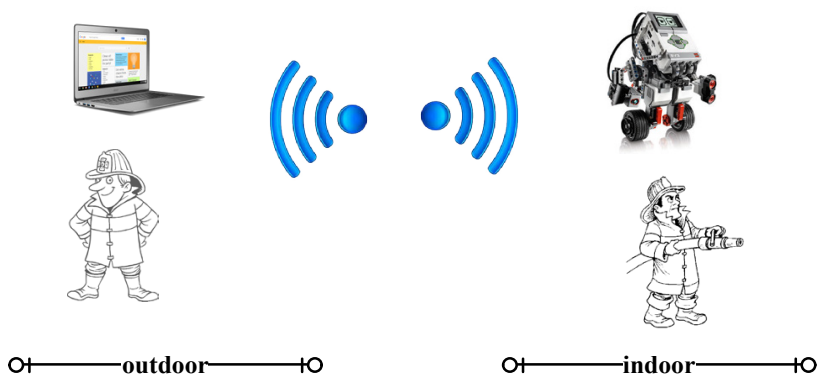

Figure 3 Hardware architecture.

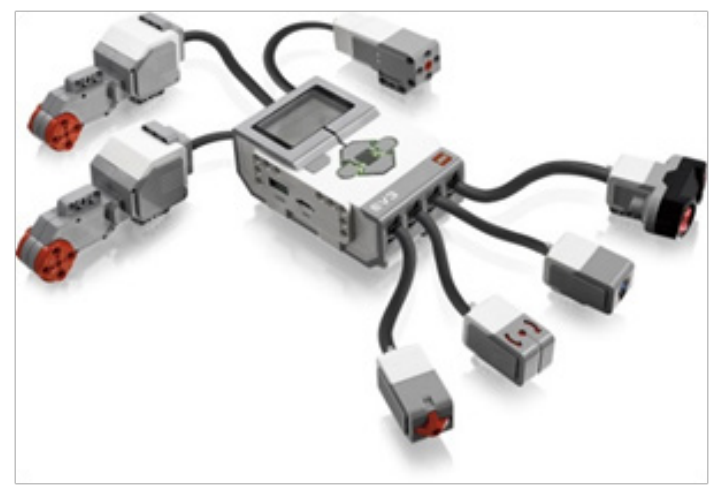

Figure 4 Lego EV3 platform.

\section{Software architecture}

The software architecture is divided into two parts: first the code runs on the Lego EV3 platform, which controls the processor, servo motors, and sensors. And second, the code runs on the remotecontrol unit, which includes displaying the robot's location, path line, obstacles position, total distance, and the shortest path table. Both systems have been implemented using $\mathrm{C \#}$ code. $\mathrm{C \#}$ has been used to implement robotic system. C\# was developed by Microsoft within its .NET initiative and later approved as a standard by Ecma. C\# is a programming language designed for common language infrastructure. The designed interface for the desktop application (remote-control unit) is presented in Figure 5, which includes a number of buttons: "Start" to begin the navigation mission, "Stop" to pause the mission, "Back" to end the mission and return to the start point, "Shortest Path" that prints all paths table in the grid named (Graph_Grid), and a Navigation textbox to show information about the total distance travelled by the robot. A WiFi connection is established between 
the robot unit and the remote-control unit, where sensors data is transmitted to the remote-control unit.

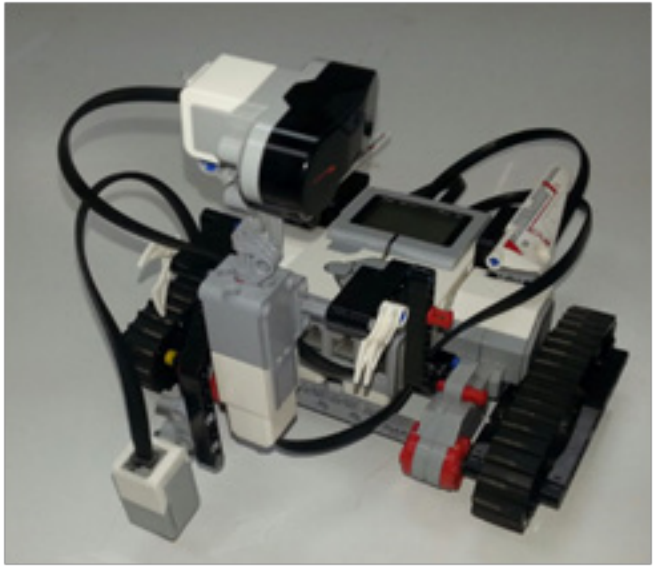

Figure 5 The designed robotic system.

\section{Real experiments}

The implemented robotic system has been tested through an environment with dimensions of $3 \times 2$ meters, where the robotic system entered the area, and then moved randomly according to the environment conditions. The travelled route of the robot from the start point is depicted in Figure 6, where the black line presents the route of the robot, and the yellow line presents the location of the robot when returning to the start point as shown in Figure 7, and the green block determines the location of the start point, and the blue blocks determines the presence and location of an object, and the red block determines the location of the pause (stop) point. Figure 8 shows the reference points with their locations which the robot has passed through.

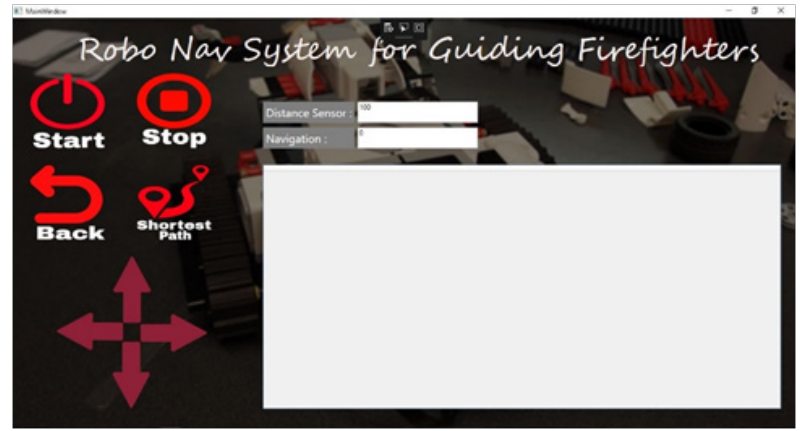

Figure 6 The Graphical User Interface for remote unit.

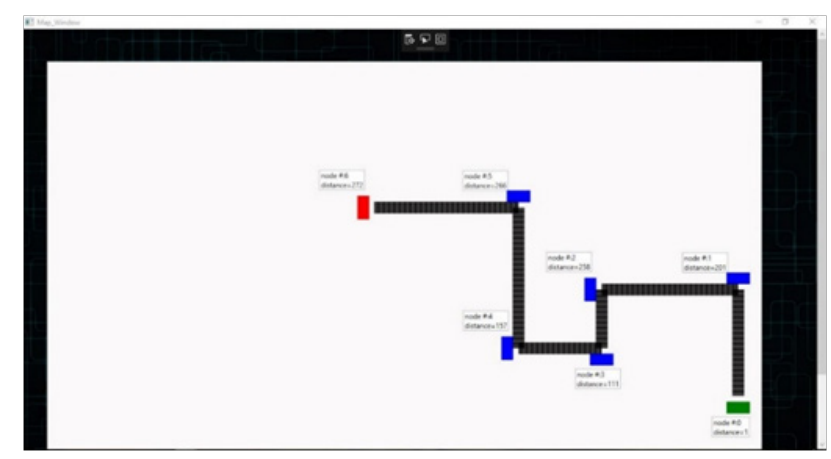

Figure 7 The travelled route of the robot (remote-control unit).

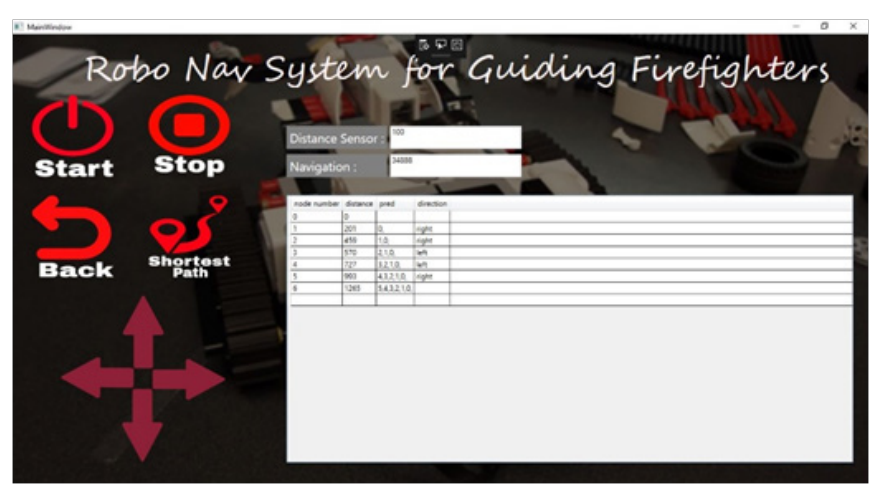

Figure 8 The remote-control form showing the distances between reference points.

\section{Conclusion \& future works}

The main objective of this work was to design, and implement a prototype robotic system, which has the ability to help firefighters in accomplishing their duties, including finding shortest path between two points (entrance and location of injured person), drawing path map with all obstacles found. The prototype system has been implemented using Lego EV3 platform, and C\# programming language to implement the code runs on the EV3 platform, and for the application run on the fire-fighter PC. For future works, we aim to use real robotic system which can afford high temperatures, and implement more functionality.

\section{Acknowledgments}

No Acknowledgement.

\section{Conflict of interest}

No conflict of interest.

\section{References}

1. Schildt S, Rottmann S, Wolf L. Communication architecture, challenges and paradigms for robotic firefighters. Proceedings of the $5^{\text {th }}$ Extreme Conference of Communication. 2013. p. 1-6.

2. Craig JJ. Introduction to robotics: mechanics and control. Upper Saddle River: Pearson Prentice Hall; 2005. p. 48-70.

3. Burke JL, Murphy RR, Rogers E, et al. Final report for the DARPA/NSF interdisciplinary study on human-robot interaction. IEEE Transactions on Systems, Man, and Cybernetics, Part C (Applications and Reviews). 2004;34(2):103-112.

4. Plackett B. Rescue Me, Robot: Machines Ready for Fire-fighting Duty. Wired Magazine. 2012.

5. Sentis L. Whole-body operational space control for locomotion and manipulation. Mechanical Engineering. 2015.

6. Shah SS, Shah VK, Mamtora P, Hapani M. Fire fighting robot. Int J Emerg Trends Technol Comp Appl. 2013;2(4):232-234.

7. Guizzo E. Rescue-robot show-down. IEEE Spectrum. 2014;51(1):52-55.

8. Xu S, Guo Y, Su W. A proof-of-concept demonstration for the transportation electrification education. Transportation Electrification Asia-Pacific (ITEC Asia-Pacific). 2014. p. 1-5.

9. Alhmiedat TA, Taleb AA, Samara G. A Prototype Navigation System for Guiding Blind People Indoors using NXT Mindstorms. IJOE. 2013;9(5):52-58. 
10. Chtourou S, Kharrat M, Amor NB, et al. Proof of concept of a cloud compilation service for robotics wireless programming. In Intelligent Systems and Informatics (SISY). 2015. p. 295-300.
11. Safaripour R, Khendek F, Glitho R, et al. A restful architecture for enabling rapid development and deployment of companion robot applications. In Computing, Networking and Communications. 2014. p. 971-976. 